\title{
Flexible and multifunctional electronics fabricated by a solvent-free and user-friendly method
}

\author{
Toan Dinh, ${ }^{* a}$ Hoang-Phuong Phan, ${ }^{a}$ Afzaal Qamar, ${ }^{a}$ Nam-Trung Nguyen,,${ }^{a}$ and Dzung Viet Dao ${ }^{a, b}$
}

Received * *2016

DOI: $10.1039 / \mathrm{b} 000000 \mathrm{x}$

Flexible and multifunctional electronic devices have been proven to show potential for various applications including human-motion detection and wearable thermal therapy. The key advantages of these systems are (1) highly stable, sensitive and fast-response devices, (2) fabrication of macroscale devices on flexible substrates, and (3) integrated (lab-on-chip) and multifunctional devices. However, their fabrication commonly requires toxic solvents, as well as time-consuming and complex processes. Here, we demonstrate the low-cost, rapid-prototyping and userfriendly fabrication of flexible transducers using recyclable, water-resistant poly(vinyl chloride) films as a substrate, and ubiquitously available pencil graphite as a functional layer without using any toxic solvents or additional catalysts. The flexible heaters showed good characteristics such as fast thermal response, good thermostability (low temperature coefficient of resistance) and low power consumption. The heaters with their capability of perceiving human motion were shown to be effective. The proof of concept of other functional devices such as vibrationbased droplet sensors and drag-force air flow sensors was also demonstrated. Results from this study indicate that a wide range of electronic devices fabricated from the environmental-friendly material by this simple and user-friendly approach could be utilized for cost-effective, flexible and low power consuming thermal therapy, health monitoring systems and other real-time monitoring devices without using any toxic chemicals or advanced processes.

\section{Introduction}

Conventional brittle materials such as silicon and silicon carbide are valuable for the development of electronic devices with high sensitivity, fast response, as well as miniaturization and integration capabilities ${ }^{1-6}$. However, alternative materials for future electronics rely on flexibility, stretchability, wearability and environment-friendliness ${ }^{7}$ for more versatile applications such as thermal therapy and thermal management ${ }^{8-14}$, health monitoring devices ${ }^{15,17-24}$ and wearable energy harvesting systems ${ }^{25}$. Recently, the research community has paid a great deal of attention to metal nanowires $8,9,12-14$, carbon nanotubes ${ }^{15,16,26}$, and conductive fibers ${ }^{20,21}$, graphene ${ }^{11,17}$ and hybrid materials ${ }^{27,28}$, which are constructed on flexible and stretchable substrates such as paper ${ }^{25,29,30}$ and polydimethylsiloxane (PDMS) ${ }^{9,15}$, because they offer excellent mechanical and electrical properties with various potential applications. For instance, recent research facilitating thermal therapy services has focused on metal nanowire-based flexible and wearable heaters owing to their excellent transparency and low voltage supply $8,9,11,13$. These heaters hold a great potential to replace conventional wraps and packs which have been employed for the treatment of human symptoms such as pain, swelling and muscle weakness $8,31,32$.

Moreover, highly flexible and stretchable train sensors have been intensively developed and applied in tactile sensing for

\footnotetext{
* Email of corresponding author: toan.dinh@griffithuni.edu.au

a Queensland Micro-and Nanotechnology Centre, Griffith University, Queensland, Australia.

${ }^{b}$ School of Engineering, Griffith University, Queensland, Australia.
}

the detection of human/robot motions ${ }^{15,17-24,33,34}$. In addition, the integration of multiple functions in a chip/device (lab-onchip) has firmly been recognized as an inevitable trend, not only for conventional electronics but also for a new generation of portable electronic devices ${ }^{35,36}$. The huge potential of these emerging wearable monitoring devices has led to their construction on thin and flexible substrates (e.g. polyethylene naphthalate (PEN) and polydimethylsiloxane (PDMS)), including power generators ${ }^{25,37,38}$ and strain sensors ${ }^{39}$.

In addition, electronic devices fabricated by conventional coating methods such as screen printing ${ }^{40}$, inkjet printing ${ }^{41}$ and spray coating ${ }^{42}$ might offer a good performance. However, these fabrication methods involved solvent-based inks consisting of toxic chemicals which can lead to various environmental issues. Furthermore, the processes also require various complex steps such as dispersion of expensive functional materials (e.g. Ag nanoparticles) in a particular solvent utilizing ultrasonication and an advanced stirring process, which are sophisticated and time-consuming. In these steps, it requires relatively high-temperature annealing process, and washing time to remove surfactants. In addition, advanced plasma treatment has been employed to improve wettability and adhesion. All above disadvantages of the conventional coating methods have limited their specific applications.

To address the challenges in the solvent-involving or complex fabrication of these electronic devices, the pen-based writing approach has been developed with increasing performance and decreasing cost. For example, ubiquitously available pencil graphite drawn on printing papers has successfully been demonstrated to be able to construct thermoresistive sen- 

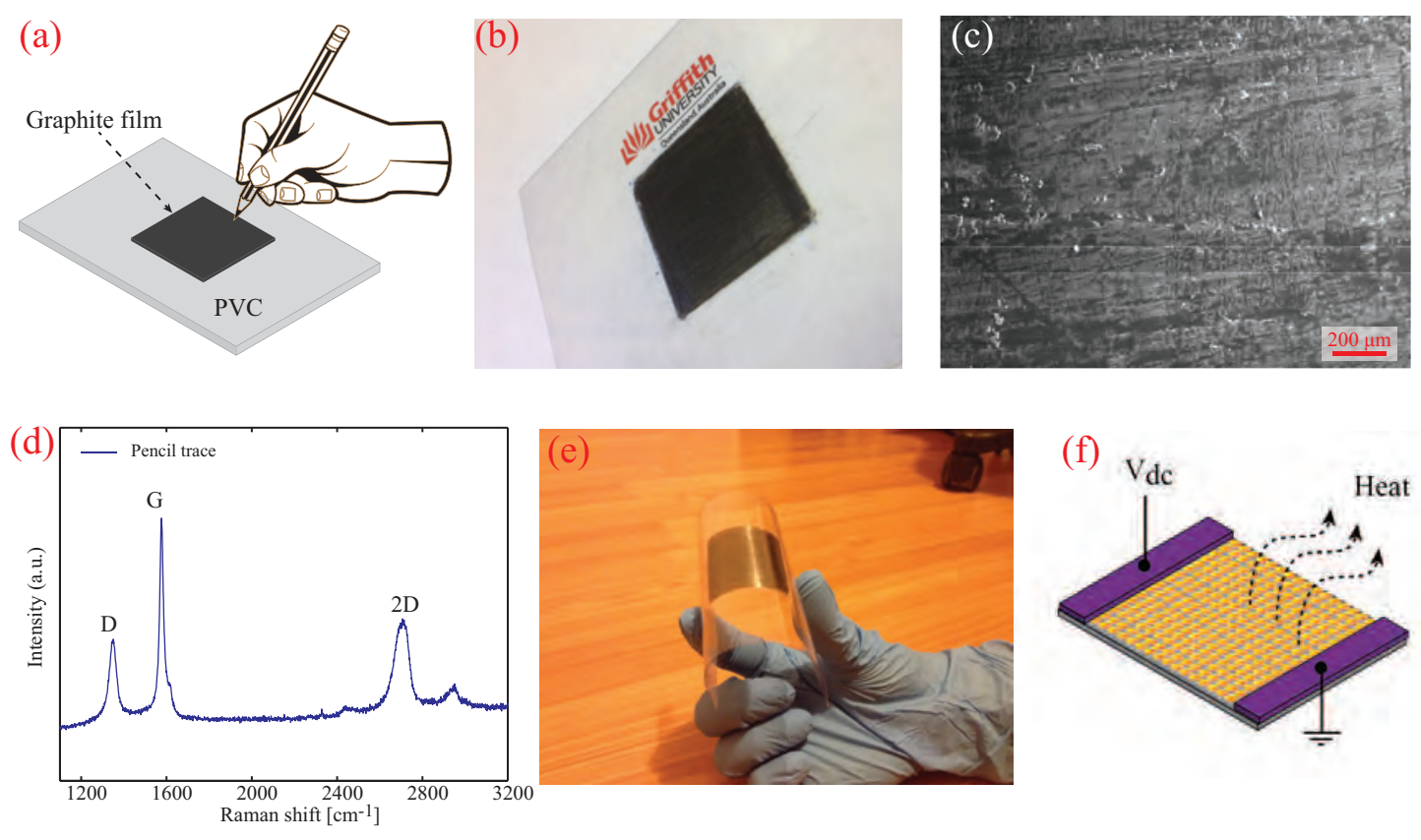

Fig. 1 GoPo material as a flexible platform for heaters. a) Schematic diagram of graphite transfer to PVC using a pencil. b) An as-drawn GoPo platform for thermal therapy applications. c) A scanning electron microscope (SEM) image of the graphite-coated PVC platform. d) Raman spectrum of the pencil trace. e) An optical image of the assembled graphite-based heater showing its outstanding flexibility. f) Schematic illustration of the as-drawn GoPo heater composed of graphite percolation network on a PVC film.

sors $^{43}$, chemiresistors ${ }^{44}$, microfluidic devices ${ }^{45,46}$, field effect transistors and filters ${ }^{47,48}$. It is true that manually drawing is a time-consuming process. However, by using, for example, a simple 2-dimensional plotter, large area deposition of pencil graphite and mass production of these devices can be achieved. The successful demonstration of these devices indicates that there is an emerging and huge demand to develop other cost-effective, eco-friendly, wearable and multifunctional electronic transducers utilizing this approach. In addition, the disadvantages of the paper-based drawn devices include the permeability of paper to moisture and gases or hydrophobic properties, henceforth constraining their utilization for specific on-site applications.

Here, we demonstrate for the first time, a simple, userfriendly, recyclable, portable and multifunctional heater for thermal therapy based on pencil graphite drawn on a poly(vinyl chloride) (GoPo) substrate without employing any toxic chemicals or complex fabrication processes. The unique properties which enhance the performance of the heater are its thermal stability with a low temperature coefficient of resistance (TCR), and fast thermal response. The TCR was found to be at least three times lower than that of the pencil-drawnon-paper devices ${ }^{43}$, and the thermal response is even faster than that of expensive and solvent-involving silver nanowire devices $^{9,11}$. The repeatability and reliability of the heaters were demonstrated by subjecting the platform to thousands of ON/OFF power cycles. The heaters can operate with a deformation of up to $\pm 0.78 \%$ and are able to monitor human motions which show a great potential for multifunctional integrated applications. In addition, the water-resistant properties of the PVC substrate were employed to successfully develop a flexible vibration-based sensor to measure the mass of a droplet. The solvent-free fabrication of drag-force flow sensors was also demonstrated with low power consuming capability. As proven through various specific novel functions, this simple but effective approach can be employed to produce low-cost, thermally stable, eco-friendly, multi-functional flexible electronic devices.

\section{GoPo materials}

Figure 1a shows a simple fabrication method to achieve a graphite film deposited on a polyvinyl chloride (PVC) substrate, using a pencil. In the drawing process, graphite particles are rubbed off due to friction between the pencil lead and the PVC substrate and then, in turn adhere to the PVC surface $^{44}$. Therefore, a pencil film can be viewed as a percolated network of ultrafine graphite particles on PVC. Figure $1 \mathrm{~b}$ shows an as-drawn square graphite film on $220 \mu$ m-thick PVC substrate, displaying the transparency of the PVC substrate. Figure 1c illustrates a scanning electron microscope (SEM) image of the pencil film, indicating its full deposition of pen- 
cil traces on the surface of the PVC substrate. A SEM image of the PVC surface is also provided in Figure 1, Supporting Information.

To confirm the quality of the graphite material created with the pencil drawing process, we examined the Raman characteristics of the pencil film. Figure 1d shows the Raman spectrum of the pencil film indicating three main prominent peaks at the wavenumbers of 1350,1580 and $2725 \mathrm{~cm}^{-1}$ corresponding to the $\mathrm{D}, \mathrm{G}$ and $2 \mathrm{D}$ bands of graphite material, respectively $46,50,51$. The D-band provides information about the number of defects and boundaries in the graphite film, and the $\mathrm{G}$ band reveals evidence of the $\mathrm{sp}^{2}$-bonded carbon networks inside the graphite film. Furthermore, the 2D peak shows an indication of stacking type in the direction perpendicular to the as-drawn film ${ }^{51}$. Since a high ratio $\left(I_{D} / I_{G}=0.34\right)$ was found for the combined $\mathrm{D}$ and $\mathrm{G}$ band intensities, this revealed a large number of defects and boundaries between graphite grains. Figure 1e indicates the flexibility of the GoPo platform with its excellent robustness under large bending, which is attributed to the elasticity of PVC. Figure 1f shows the heating principle of the graphite percolated network. As such, when a constant voltage $V_{d c}$ is applied, heat or temperature rise occurs owing the resistive properties of the graphite film. In addition, the linear current-voltage characteristic of the GoPo platform was observed (Figure 2, Supporting Information) and indicated that this material could be used as a resistive element for Joule heating-based heaters.

\section{Flexible and wearable heaters for ther- motherapy}

For thermal therapy applications, the following characteristics of a heater are required: (1) ability to operate under a low supply voltage/power, (2) ability to sufficiently raise temperatures, (3) to have fast thermal responses, (4) to have long-term stability and (5) demonstrated wearability. Therefore, the thermal performance of GoPo was investigated as shown in Figure 2. Owing to the resistive properties of the graphite film, a constant voltage of 5,10 , and $15 \mathrm{~V}$ was applied at two electrodes within a time frame of $80 \mathrm{~s}$, to raise the temperature of the heater (Figure 2a). It is evident that at a low applied voltage of $10 \mathrm{~V}$, the heater temperature can reach a steady-state temperature of approximately $50{ }^{\circ} \mathrm{C}$, which is suitable for thermal therapy. The temperature of the reverse side of the heater was also found to be approximately equal to that of the front surface (the surface with deposited graphite film). This result confirms that the heat could be effectively transferred to the human skin when the heater is employed for wearable therapy. In addition, since the glass transition temperature of PVC is approximately $80{ }^{\circ} C^{52}$, the GoPo platform could be suitable for the thermal treatment with the required temperatures normally lower than $50{ }^{\circ} \mathrm{C}$.

Figure $2 \mathrm{~b}$ indicates a linear relationship between the steadystate temperature and the power consumption of the heater.
A low power consumption of only $0.65 \mathrm{~W}$ was observed to reach a temperature of $70{ }^{\circ} \mathrm{C}$, which was comparable to and even lower than that of various other heaters for wearable applications ${ }^{8,9}$. The corresponding heat flux on each side of the graphite film was $1040 \mathrm{~W} / \mathrm{m}^{2}$. Using Newton's law of cooling under the assumption that the convective heat transfer was dominant compared to heat conduction and heat radiation, the average heat transfer coefficient was found to be approximately $20.8 \mathrm{~W} / \mathrm{m}^{2} \mathrm{~K}$. This is comparable to the conventional heat transfer coefficients for natural convection in gases ${ }^{53}$.

For practical applications, the fast thermal response of a heater is an important parameter which can be attributed to the thickness of the heater substrate. Theoretical and experimental studies have shown that the thinner the substrates, the faster the time response of the heater ${ }^{9,11}$. By utilizing the 220 $\mu \mathrm{m}$ thick PVC substrate, we have confirmed that a response time of approximately 9 s can be achieved (Figure 3, Supporting Information) which is comparable to and even fater than that of advanced stretchable heaters using silver nanowire on $\mathrm{PDMS}^{9}$. This response time is faster than that of various expensive substrate materials such as graphene on polyethylene naphthalate (PEN) ${ }^{11}$ and Ag NW on a ultra-thin glass ${ }^{54}$. This fast response is also attributed to the efficient heat transfer to the polymer substrate and low volumetric heat capacity of $\mathrm{PVC}^{55}$.

When the heating power compensates for the heat loss at the heater interfaces, a high steady-state temperature is maintained and may lead to the degradation of the heater performance. Therefore, it is important to evaluate the long-term thermal repeatability of the heater. Figure $2 \mathrm{c}$ indicates that no significant change in heating performance has been observed after 1000 cycles of the ON (10 V, $60 \mathrm{~s})$ and OFF (0 V, $30 \mathrm{~s})$ power, and therefore the heater satisfies the reliability requirements for practical applications such as thermal therapy.

The inset table in Figure 2b suggests that the heater has a steady resistance of approximately $351 \Omega$, which agrees with the value of the heater resistance recorded through the Joule heating process, as illustrated in Figure $2 \mathrm{~d}$. The resistance changes were approximately $2 \%$ when a voltage of $10 \mathrm{~V}$ was applied and a temperature of $50{ }^{\circ} \mathrm{C}$ was reached. Figure $2 \mathrm{e}$ shows a good thermally-stable characteristic of the GoPo platform. The temperature coefficient of resistance (TCR) defined by the relative resistance change to temperature variation ${ }^{56}$, was found to be approximately $900 \mathrm{ppm} / \mathrm{K}$ (Figure 2e) for temperatures up to $50{ }^{\circ} \mathrm{C}$. Thus, Figures $2 \mathrm{a}$ to e show that the graphite film has stable thermal properties.

It is believed that the thermal response of the GoPo platform (Figure 2e) is attributed to the large thermal expansion of the polymer substrate ${ }^{57,58}$ (thermal expansion coefficient of $\sim 1.2 \times 10^{-4} K^{-1}$ ) with increasing temperatures, which leads to the positive value of TCR. The expansion of the substrate caused the increase in the electrical resistance of the platform due to the piezoresistive effect of graphite which has been reported in literature ${ }^{29,44,49}$. The increase of the temperature 

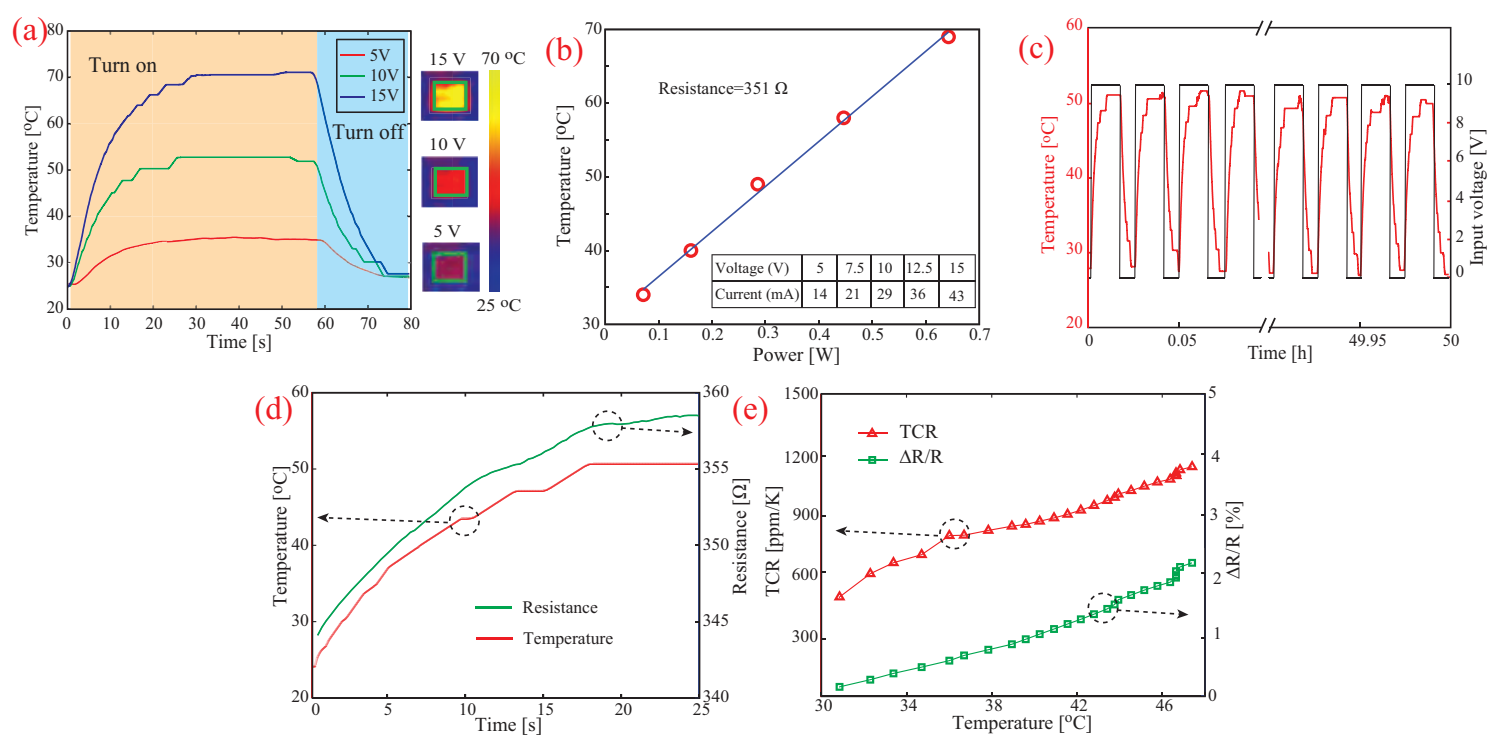

Fig. 2 Electrical heating characteristics of the heater. a) Temperature profile of the GoPo heater for different applied DC voltages. b) Temperature as a function of power consumption for the heater. The inset table shows the current-voltage relationship. c) ON/OFF response of the film heater under 1000 cycles showing the long-term stability of the heater. d) Electric heating behaviour of the GoPo heater measured as a change in electrical resistance and temperature under an applied DC voltage of $10 \mathrm{~V}$. e) Thermally-stable characteristics of the heater with low temperature coefficient of resistance (TCR $\sim 900 \mathrm{ppm})$.
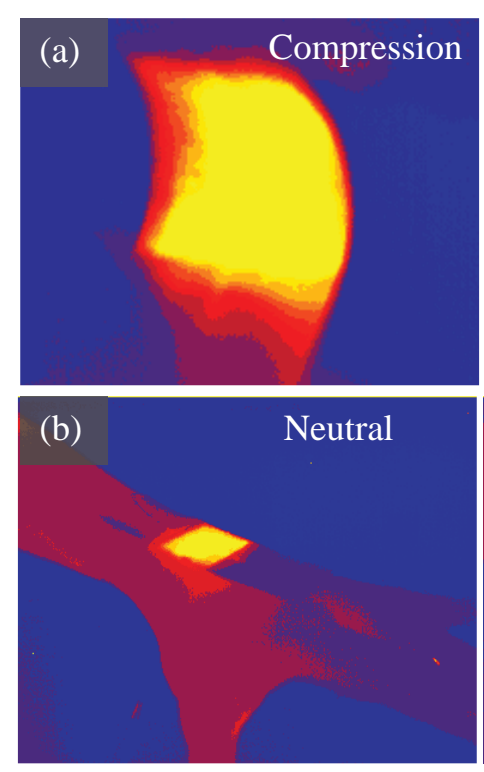
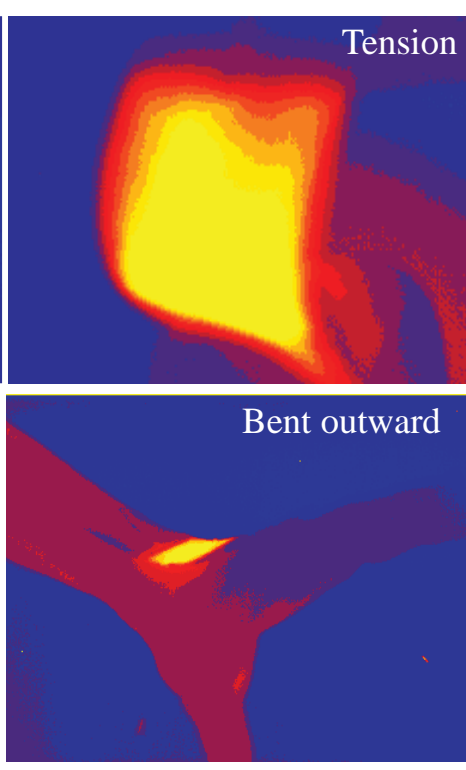
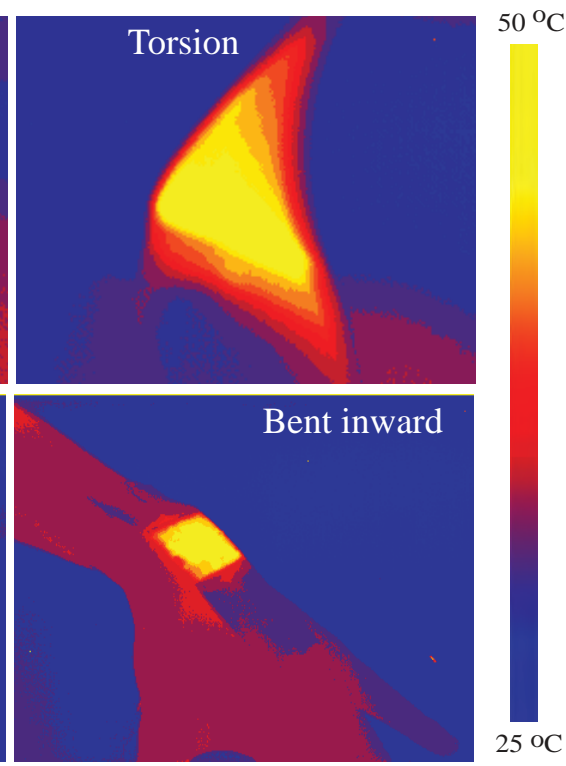

Fig. 3 Flexible and wearable heater demonstration. a) Infrared pictures of the graphite-based flexible heater under mechanical-stress conditions: compression (left), tension (centre) and torsion (right). (b) Temperature distribution images of the wearable GoPo platform affixed to human twist at neutral (left), outward bending (centre), and inward bending (right).

could also lead to a decrease in the graphite resistivity due to the contribution of the thermionic emission current ${ }^{43}$. However, the TCR of the GoPo platform was observed to be small because of the contribution of both thermal expansion of PVC and the thermally activated conduction of graphite. This thermal stability is valuable in developing other functions such as the measurement of human motions at a body temperature of approximately $37^{\circ} \mathrm{C}$.

As thermal therapy devices are usually attached to human skin, flexibility is an essential factor for the heater. The underlying GoPo platform is flexible and lightweight. In order to test its functionality under typical in-service loading con- 

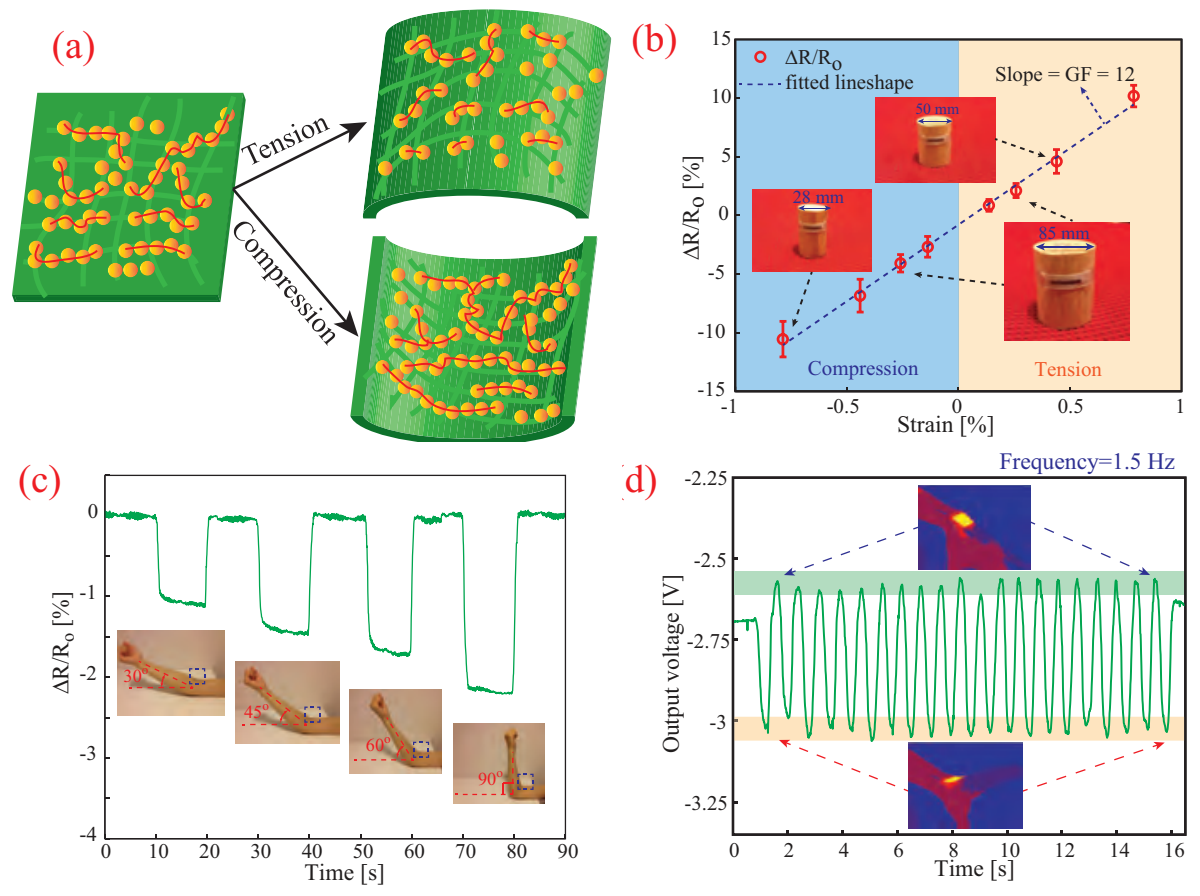

Fig. 4 GoPo platform functions as a flexible strain gauge. a) Schematic drawing shows that the number of connected graphite particle chains varies depending on the types of mechanical deformation. b) Electrical resistance changes under compression and tension up to $0.78 \%$, indicating a gauge factor of approximately 12. c) Motion response of the sensor under different bending angles of human arm (the insets). d) Dynamic response of the output voltage from four-point measurement of the heater affixed to human twist (frequency $=1.5 \mathrm{~Hz}$ ).

ditions, the heater was bent under compression, tension and twisting. Figure $3 \mathrm{a}$ indicates that the heater is mechanically robust yet bendable and wearable due to the flexible nature of PVC. In addition, as PVC is bio-compatible and has no adverse effects on human skin and the immune systems ${ }^{59,60}$, we have demonstrated the feasibility of using GoPo for wearable thermal therapy by directly affixing the heater to a human wrist and observing its operation under various physical disturbances such as bending and twisting. Figure $3 \mathrm{~b}$ shows the temperature distribution of the heater under three different operating conditions including bent outward, neutral and bent upward. The Supplementary Movie 1 (Supporting Information) validates the operation of the heater under these conditions. In addition, no significant temperature change has been observed after 500 cycles bent outward and upward, which indicates a long term stability of the heater under bending conditions.

The low supplied voltage and power, fast thermal response, excellent thermostability, as well as the flexibility and biocompatibility of the heater have proven the feasibility of using GoPo for thermal treatment purposes.

\section{Strain sensors for human-motion detection}

Apart from the capacity for forming flexible heating elements for thermal therapy, the GoPo platform also holds a potential for making wearable devices to perceive human motions because of its thermal stability having no significant temperature-effect on electrical resistance of the platform when attached to the human body. The following section discusses the strain characteristics of the GoPo platform and demonstrates its strain-sensing applications. The principle of a wearable GoPo strain sensor is based on the piezoresistance of the graphite layer as shown in Figure 4a. The principle of a wearable GoPo strain sensor is based on the piezoresistance of the graphite layer as shown in Figure 4a. The idea of the piezoresistance in the graphite trace is that the deformations of a graphite trace can lead to a change in the number of connected graphite chains. As such, any outward tension in the graphite trace can stretch and effectively separate the graphite particle network, leading to a decrease in the number of graphite chains; hence there would be an electrical resistance increase in the graphite film. However, a compression can tighten the particle network and increase the number of graphite chains, resulting in a decrease in the electrical resistance $^{44}$.

Figure 4b shows the strain characteristics of the GoPo platform with the strain range from $-0.78 \%$ to $+0.78 \%$, which is obtained by wrapping the GoPo sensors around cylinders with different diameters. The applied strain $\varepsilon$ can be calculated as $\varepsilon= \pm t / 2 r$, where $t$ is the thickness of the PVC and $r$ is the radius of the cylinders. To estimate the sensitivity of GoPo, the gauge factor (GF) was used and expressed as 

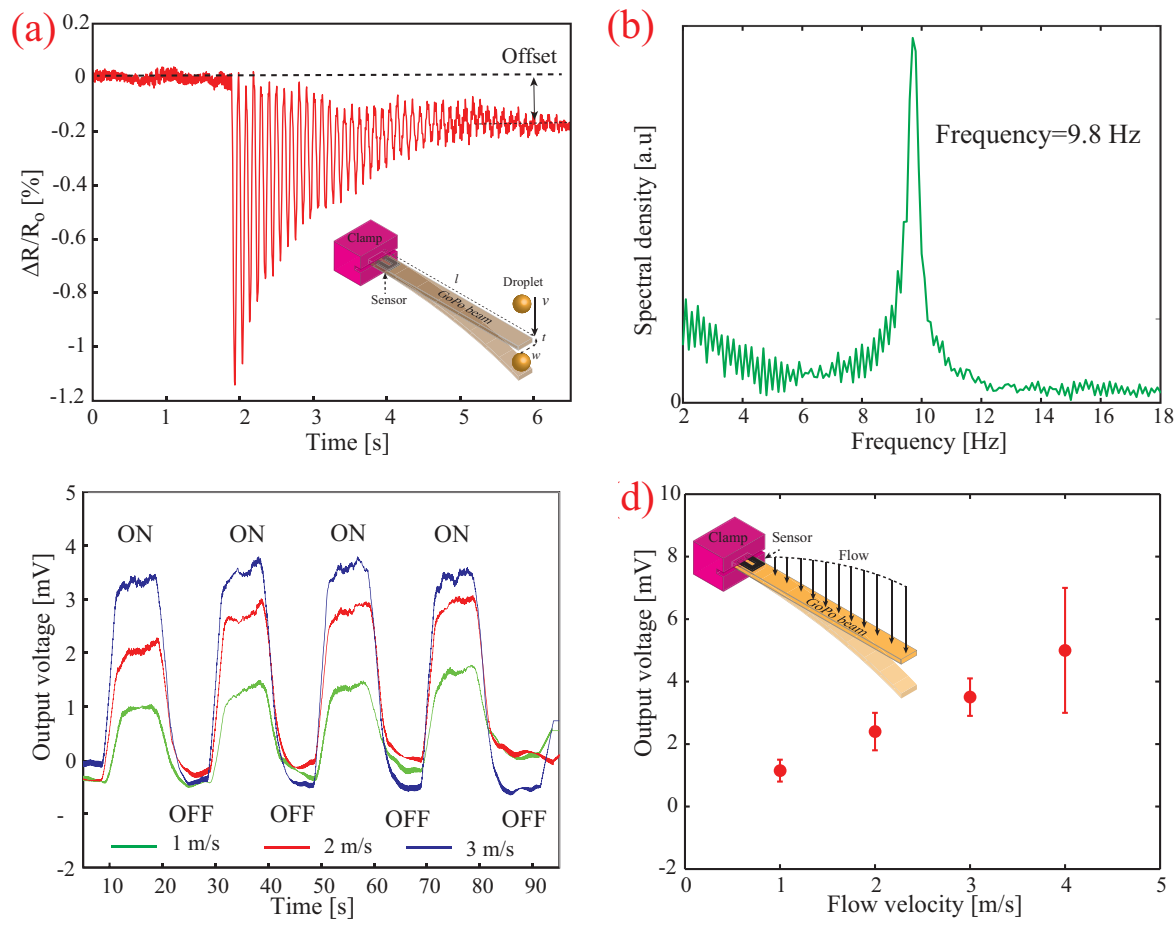

Fig. 5 Resonant-based mass sensors and drag-force flow sensors. a) Time dependent intensity of the oscillations of a water droplet; the inset shows the schematic diagram of a vibration cantilever under the insertion of a droplet. b) The spectral density obtained by Fourier transforming the data in the panel (a), indicating a resonant frequency of $9.8 \mathrm{~Hz}$. c) Responses of the flow sensor under different flow velocities. d) The performance of the flow sensor; the inset shows the schematic diagram of flow-induced displacement of the cantilever.

$G F=\Delta R / R \times 1 / \varepsilon$, where $\Delta R / R$ is the relative change of resistance and $\varepsilon$ is the mechanical strain applied to the sensor. A linear electrical resistance change was observed with applied strain, corresponding to a gauge factor of approximately 12 , which was six times higher than that of most metals $(\sim 2)$, and also even better than recently reported advanced graphene and carbon nanotube based strain sensors. This result indicates that the GoPo platform can serve as a strain-sensing element. The long term stability of the GoPo sensors under applied stresses was also observed (Figure 4, Supporting Information).

Based on the above mechanical sensing properties of GoPo, we demonstrated its potential for wearable strain sensors which can detect human motion. We assembled a graphite sensor on a human arm and examined the dynamic monitoring of the arm bending movements. Figure $4 \mathrm{c}$ shows a realtime response of the sensor under different angles $\left(30^{\circ}, 45^{\circ}\right.$, $60^{\circ}, 90^{\circ}$ ), which showed an increase in the output voltage with increasing bending angle and reversible characteristics after returning to the initial position $\left(0^{\circ}\right)$. The rapid response and repeatability of the strain sensors with their capability of working in different environments (e.g. in air and under water) were observed, which confirms the possibility of using GoPo to detect the human motion (Figure 5, Supporting Information). We also demonstrated the integration of the wearable heater for human motion detection using a four-point measurement method with a large output signals. Details of the measurement setup are provided in Figure 6, Supporting Information. The inward and outward bending motions of the human twist at a frequency of $1.5 \mathrm{~Hz}$ were monitored as shown in Figure $4 \mathrm{~d}$. The average output voltage change of up to $0.5 \mathrm{~V}$ was observed, indicating that the motion at the injury joint of patients can be recorded to evaluate its impacts to the efficiency of thermal treatment process. The above results confirm the suitability of utilizing GoPo for multifunctional wearable applications.

\section{Mass sensors and flow sensors}

We also demonstrated that GoPo can be employed for lowcost portable mechanical sensors such as mass sensors and flow sensors. As mentioned above, the demonstrations have showed the motion signal frequency of up to $1.5 \mathrm{~Hz}$, corresponding to a time response of approximately $700 \mathrm{~ms}$, and thus GoPo holds promise for high frequency and fast time response mechanical sensors. Figure 5a exhibits the time dependent vibrations of the strain sensor induced by the water droplet, which indicates a damped vibration. In principle, the natural resonance frequency of the cantilever does not depend on the excitation method (e.g. droplet falling). On one hand, 
a very small falling height can lead to a small vibration amplitude. On the other hand, a high falling height can result in a large variance of the impact location, and even spillage at high impact speeds ${ }^{64}$. Both of above cases cause an inaccurate measurement of the droplet mass added to the cantilever. Therefore, in the vibration measurement, an appropriate height $(\sim 50 \mathrm{~mm})$ was employed to obtain the time response shown in Fig. 5a. The vibrational frequency of the droplet on the GoPo cantilever was found to be $9.8 \mathrm{~Hz}$ (Figure $5 b$ ), using Fourier transformation. Consequently, a response time of approximately $50 \mathrm{~ms}$ was obtained, which was comparable with the response time of nanowire devices ${ }^{61}$, and even faster than that of the various devices with response time ranging from 100 to $3800 \mathrm{~ms}^{62,63}$. The damping ratio was determined from the experimental data by calculating the logarithmic decay, which depended on the amplitude of vibration and the number of cycles between peaks. The damping ratio value was calculated to be approximately $1.2 \%$ corresponding to only $50 \mathrm{ppm}$ difference between the natural resonant frequency and the damped frequency, which can be neglected. The resonant frequency of the cantilever with a mass at the free end is estimated as ${ }^{64} \omega=\beta^{2} \sqrt{\frac{E I}{m_{\text {beam }} l+m_{\text {drop }}}} \frac{1}{L^{3 / 2}}$, where $m_{\text {beam }}$ and $m_{\text {drop }}$ are the beam mass per unit length and the mass of the droplet, respectively. $\beta$ is a pre-factor. $E, I, L$ are the Young modulus, inertial moment, and distance from the fixed end of the PVC cantilever to the centre of the droplet, respectively. The relative position of the cantilever and the syringe was carefully fixed to make sure the droplet impacts 3 $\mathrm{mm}$ from the free end of the cantilever to avoid edge spilling during impact. Moreover, ambient air currents are minimized to reduce the deviation of impact location. In addition, considering an impact location of $3 \pm 0.5 \mathrm{~mm}$ between the droplet and the free end of the cantilever, the mass of the droplet was estimated to be $0.033 \pm 0.003 \mathrm{~g}$. The high mass resolution and fast response time indicates the feasibility of using GoPo for mass sensors, vibration measurements and various real-time observing systems including health monitoring and wearable devices, and robotic sensors.

The GoPo platform can also be used as a flow sensor as shown in Figure 5c. The experimental setup and other conditions related to the flow testing are described in detail in Figure 7, Supporting Information. The flexibility of the GoPo sensor was able to provide sufficient displacement, and then stress and strain to detect the drag force generated from the range of flow rates of 1 to $4 \mathrm{~m} / \mathrm{s}$. The output voltage from the sensor increased with the increasing flow velocity (Figure 5d), and was found to be proportional to velocity of air flow with a sensitivity of $1.25 \mathrm{mV} / \mathrm{m} / \mathrm{s}$. This indicates the possibility of using GoPo for sensitive air flow sensors.

\section{Conclusion}

In conclusion, the results presented herein demonstrate the simple and solution-free fabrication of the economical, multifuntional and environment-friendly wearable therapy systems with thermally stable properties, low power consumption and fast thermal response. The heaters also functioned as a highly sensitive human-motion sensing device. The successful demonstration of the high-resolution droplet mass sensor indicated the obvious advantage of using a polymeric substrate for motion sensing in wet conditions. Other functions such as drag-force air flow sensors were also developed with low energy consumption. We believe that the devices constructed by this solvent-free, user-friendly and rapid prototyping approach are evidence for wearable electronic devices which find various practical applications in thermal therapy, healthcare and real-time monitoring systems with high performance and low cost.

\section{Experimental Section}

Material: 8B-type pencil (Faber-Castell) was used to draw graphite films on $220 \mu \mathrm{m}$-thick PVC (A4, PVC blinding covers) which was roughened using emery papers. The area where the pencil graphite would be deposited was manually roughened with an average applied force of $2 \mathrm{~N}$. The electrical interconnects were formed utilizing the high conductivity silver paste (1863616, RS Components).

Heaters and sensors: Square heaters with dimensions of $2.5 \mathrm{~cm} \times 2.5 \mathrm{~cm}$ were fabricated, and the strain sensors were constructed by the 15 -time drawing of pencil traces $(3 \mathrm{~mm} \times$ $40 \mathrm{~mm}$ ). The strain sensor was covered with a thin layer of an insulation epoxy (Araldite Super strength Epoxy, Selleys) to demonstrate the motion detection under water. First, A shadow mask was attached to the GoPo platform. An insulation epoxy layer was then coated on the electrodes and asdrawn graphite sensing layers. Finally, the devices were dried on hot plate at $60{ }^{\circ} \mathrm{C}$ for 1 hour. In addition, the U-shaped strain sensing element (longitudinal length of $3 \mathrm{~mm}$, transverse length of $1 \mathrm{~mm}$ and trace width of $1 \mathrm{~mm}$ ) was drawn on a PVC substrate and then the cantilever was shaped using a laser cutter (Speedy300TM, Trotecs) with a dimension of $55 \mathrm{~mm} \times 3 \mathrm{~mm} \times 0.22 \mathrm{~mm}$. This cantilever was used as the mass sensor and flow sensor.

Characterisation: All time-dependent temperature response, thermal images and wearable thermal heaters were taken by an infrared camera (GOBI-1513, Xenics Infrared Solutions). Droplets were generated from a syringe, freely dropped with an acceleration of the earth and then attached on the top-end of the cantilever. The time dependent intensity variations measured by the GoPo cantilever were recorded using an oscillator (MSO-X 3104A, Agilent Technologies) and analysed using Matlab (Mathworks). The data were collected 
with sampling frequency of $1 \mathrm{kHz}$. An air blower (LB0115002, Industrial Equipment and Control) generated air flow ranging from 1 to $4 \mathrm{~m} / \mathrm{s}$ at room temperature $\left(23{ }^{\circ} \mathrm{C}\right)$ for flow sensor characterization.

\section{Acknowledgements}

This work was performed in part at the Queensland node of the Australian National Fabrication Facility, a company established under the National Collaborative Research Infrastructure Strategy to provide nano and micro-fabrication facilities for Australia's researchers. This work has been partially supported by the Griffith University's New Researcher Grants and Australian Research Council grant LP150100153. The authors would like to thank Dr Peter Woodfield for his valuable comments on the manuscript.

\section{References}

1 Y. Cui, C. M. Lieber, Science, 2001, 291, 851.

2 M. Schulz, Nature, 1999, 399, 729.

3 H.P. Phan, D.V. Dao, K. Nakamura, S. Dimitrijev and N.T. Nguyen, JMEMS, 2015, 24, 1663.

4 A. Vatani, P. L. Woodfield, D. V. Dao, Journal of Molecular Liquids, 2015, $211,712$.

5 T. Dinh, D. V. Dao, H.-P. Phan, L. Wang, A. Qamar, N.-T. Nguyen, P. Tanner, M. Rybachuk, Applied Physics Express, 2015, 8, 061303.

6 T. Dinh, H.-P. Phan, T. Kozeki, A. Qamar, T. Namazu, N.-T. Nguyen, D. V. Dao, RSC Advances, 2015, 5, 106083.

7 N.-T. Nguyen, Micro and Nanosystems, 2015, 7, 2.

8 S. Choi, J. Park, W. Hyun, J. Kim, J. Kim, Y. B. Lee, C. Song, H. J. Hwang, J. H. Kim, T. Hyeon, ACS nano, 2015, 9, 6626.

9 S. Hong, H. Lee, J. Lee, J. Kwon, S. Han, Y. D. Suh, H. Cho, J. Shin, J. Yeo, S. H. Ko, Advanced materials, 2015 , 27,4744 .

10 D. Kim, L. Zhu, D.-J. Jeong, K. Chun, Y.-Y. Bang, S.-R. Kim, J.-H. Kim, S.-K. Oh, Carbon, 2013, 63, 530.

11 J. Kang, H. Kim, K. S. Kim, S.-K. Lee, S. Bae, J.-H. Ahn, Y.-J. Kim, J.-B. Choi, B. H. Hong, Nano letters, 2011, 11, 5154.

12 J. Li, J. Liang, X. Jian, W. Hu, J. Li, Q. Pei, Macromolecular Materials and Engineering, 2014, 299, 1403.

13 C. Celle, C. Mayousse, E. Moreau, H. Basti, A. Carella, J.-P. Simonato, Nano Research, 2012, 5, 427.

14 P.-C. Hsu, X. Liu, C. Liu, X. Xie, H. R. Lee, A. J. Welch, T. Zhao, Y. Cui, Nano letters, 2014, 15, 365.

15 T. Yamada, Y. Hayamizu, Y. Yamamoto, Y. Yomogida, A. Izadi-Najafabadi, D. N. Futaba, K. Hata, Nature nanotechnology, 2011, 6, 296.

16 V. T. Dau, D. V. Dao, T. Yamada, B. T. Tung, K. Hata, S. Sugiyama, Smart Materials and structures, 2010, 19, 075003.
17 J. Yang, D. Wei, L. Tang, X. Song, W. Luo, J. Chu, T. Gao, H. Shi, C. Du, RSC Advances, 2015, 5, 25609.

18 D. H. Kim, S. Wang, H. Keum, R. Ghaffari, Y. S. Kim, H. Tao, B. Panilaitis, M. Li, Z. Kang, F. Omenetto, Small, 2012, 8, 3263.

19 J. Kim, M. Lee, H. J. Shim, R. Ghaffari, H. R. Cho, D. Son, Y. H. Jung, M. Soh, C. Choi, S. Jung, Nature communications, 2014, 5.

20 W. Zeng, L. Shu, Q. Li, S. Chen, F. Wang, X. M. Tao, Advanced Materials, 2014, 26, 5310.

21 S. Lee, S. Shin, S. Lee, J. Seo, J. Lee, S. Son, H. J. Cho, H. Algadi, S. AlSayari, D. E. Kim, Advanced Functional Materials, 2015.

22 X. Zhao, Q. Hua, R. Yu, Y. Zhang, C. Pan, Advanced Electronic Materials, 2015.

23 S. G. Yoon, H.-J. Koo, S. T. Chang, ACS applied materials \& interfaces, 2015.

24 R. Matsuzaki, K. Tabayashi, Advanced Functional Materials, 2015.

25 S. S. Won, M. Sheldon, N. Mostovych, J. Kwak, B.-S. Chang, C. W. Ahn, A. I. Kingon, I. W. Kim, S.-H. Kim, Applied Physics Letters, 2015, 107, 202901.

26 S. Yao, Y. Zhu, Advanced Materials, 2015, 27, 1480.

27 H. K. Park, S. M. Kim, J. S. Lee, J.-H. Park, Y.-K. Hong, C. H. Hong, K. K. Kim, Synthetic Metals, 2015, 203, 127.

28 S. Zhu, J. H. So, R. Mays, S. Desai, W. R. Barnes, B. Pourdeyhimi, M. D. Dickey, Advanced Functional Materials, 2013, 23, 2308.

29 X. Liao, Q. Liao, X. Yan, Q. Liang, H. Si, M. Li, H. Wu, S. Cao, Y. Zhang, Advanced Functional Materials, 2015, 25, 2395.

30 Q. Zhong, J. Zhong, X. Cheng, X. Yao, B. Wang, W. Li, N. Wu, K. Liu, B. Hu, J. Zhou, Advanced Materials, 2015, 27, 7130 .

31 S. Michlovitz, L. Hun, G. N. Erasala, D. A. Hengehold, K. W. Weingand, Archives of physical medicine and rehabilitation, 2004, 85, 1409.

32 J. M. Mayer, L. Ralph, M. Look, G. N. Erasala, J. L. Verna, L. N. Matheson, V. Mooney, The Spine Journal, 2005, 5, 395.

33 X. Liao, Z. Zhang, Q. Liao, Q. Liang, Y. Ou, M. Xu, M. Li, G. Zhang and Y. Zhang, Nanoscale, 2016.

34 Q. Liao, M. Mohr, X. Zhang, Z. Zhang, Y. Zhang and H.J. Fecht, Nanoscale, 2013, 5, 12350.

35 R. Jain, D. Li, M. Gupta, L. J. Manderfield, J. L. Ifkovits, Q. Wang, F. Liu, Y. Liu, A. Poleshko, A. Padmanabhan, Science, 2015, 348, aaa6071.

36 X. Liao, Q. Liao, Z. Zhang, X. Yan, Q. Liang, Q. Wang, M. Li and Y. Zhang, Advanced Functional Materials, 2016.

37 F. Wang, Y.-W. Mai, D. Wang, R. Ding, W. Shi, Sensors and Actuators A: Physical, 2015, 233, 195.

38 Y.-K. Fuh, P.-C. Chen, Z.-M. Huang, H.-C. Ho, Nano Energy, 2015, 11, 671. 
39 Y. Qin, Q. Peng, Y. Ding, Z. Lin, C. Wang, Y. Li, F. Xu, J. Li, Y. Yuan, X. He, ACS nano, 2015, 9, 8933.

40 R. Gupta, S. Walia, M. Hosel, J. Jensen, D. Angmo, F.C. Krebs and G.U. Kulkarni, Journal of Materials Chemistry A, 2014, 2, 10930.

41 J. Kang, Y. Jang, Y. Kim, S.H. Cho, J. Suhr, B.H. Hong, J.B. Choi and D. Byun, Nanoscale, 2015, 7, 6567.

42 T. Akter, J. Joseph and W.S. Kim, IEEE electron device letters, 2012, 33, 902.

43 T. Dinh, H.-P. Phan, D. V. Dao, P. Woodfield, A. Qamar, N.-T. Nguyen, Journal of Materials Chemistry C, 2015, 3, 8776.

44 C.-W. Lin, Z. Zhao, J. Kim, J. Huang, Scientific reports, 2014, 4.

45 N. Kurra, G. U. Kulkarni, Lab on a Chip, 2013, 13, 2866.

46 N. Dossi, R. Toniolo, A. Pizzariello, F. Impellizzieri, E. Piccin, G. Bontempelli, Electrophoresis, 2013, 34, 2085.

47 N. Kurra, D. Dutta, G. U. Kulkarni, Physical Chemistry Chemical Physics, 2013, 15, 8367.

48 S. Kanaparthi and S. Badhulika, Green Chemistry, 2016.

49 H.-P. Phan, D. V. Dao, T. Dinh, H. Brooke, A. Qamar, N.T. Nguyen, Y. Zhu, 28th IEEE International Conference on Micro Electro Mechanical Systems (MEMS), 2015.

50 F. Tuinstra, J. L. Koenig, The Journal of Chemical Physics, 1970, 53, 1126.

51 M. Pimenta, G. Dresselhaus, M. S. Dresselhaus, L. Cancado, A. Jorio, R. Saito, Physical chemistry chemical physics, 2007, 9, 1276.

52 C. E. Wilkes, PVC Handbook. Hanser Verlag., 2005, ISBN 1-56990-379-4.

53 T. L. Bergman, F. P. Incropera, A. S. Lavine, Fundamentals of heat and mass transfer, John Wiley \& Sons, 2011.

54 S. Ji, W. He, K. Wang, Y. Ran, C. Ye, small, 2014, 10, 4951.

55 A. Mathur, A. Srivastava, G.D. Agrawal, S. Mathur and J. Mathur, Energy and Buildings, 2015, 87, 37.

56 T. Dinh, H.-P. Phan, T. Kozeki, A. Qamar, T. Fujii, T. Namazu, N.-T. Nguyen and D. V. Dao, Materials Letters, 2016, $177,80$.

57 R. Simha, P. S. Wilson, Macromolecules, 1973, 6, 908.

58 A. Bessonov, M. Kirikova, S. Haque, I. Gartseev and M. J. Bailey, Sensors and Actuators A: Physical, 2014, 206, 75.

59 S. Mallakpour, M. Javadpour, Colloid and Polymer Science, 2015, 293, 2565.

60 J. Yianni, Medical device technology, 1995, 6, 20.

61 S. Gong, W. Schwalb, Y. Wang, Y. Chen, Y. Tang, J. $\mathrm{Si}$, B. Shirinzadeh, W. Cheng, Nat. Commun., 2014, 5 , 3132 .

62 Z. Wang, J. Qi, X. Yan, Q. Zhang, Q. Wang, S. Lu, P. Lin, Q. Liao, Z. Zhang, Y. Zhang, RSC Adv., 2013, 3, 17011.

63 S. Harada, W. Honda, T. Arie, S. Akita, K. Takei, ACS nano, 2014, 8, 3921.

64 S. Gart, J.E. Mates, C.M. Megaridis and S. Jung, Physical Review Applied, 2015, 3, 044019. 